Research Paper

\title{
Signature of gene aberrant alternative splicing events in pancreatic adenocarcinoma prognosis
}

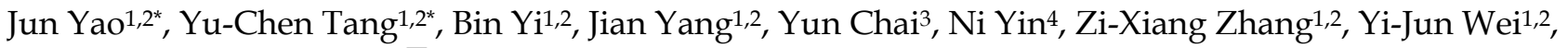 \\ De-Chun Li ${ }^{1,2}$, Jian Zhou ${ }^{1,2 \bowtie}$
}

1. Department of General Surgery, the First Affiliated Hospital of Soochow University, Suzhou, Jiangsu, 215006, China.

2. Pancreatic Disease Research Centre, The First Affiliated Hospital of Soochow University, Suzhou, Jiangsu, 215006, China.

3. Department of Plastic Surgery, Suzhou Municipal Hospital, Suzhou, Jiangsu, 215006, China.

4. Department of Oncology, the First Affiliated Hospital of Soochow University, Suzhou, Jiangsu, 215006, China.

* Jun Yao and Yu-Chen Tang contributed equally to this work.

$\square$ Corresponding author: Jian Zhou, Department of General Surgery, the First Affiliated Hospital of Soochow University, No. 188, Shizi Road, Suzhou, 215006 Jiangsu, China; E-mail: zhoujian06@suda.edu.cn

(c) The author(s). This is an open access article distributed under the terms of the Creative Commons Attribution License (https://creativecommons.org/licenses/by/4.0/). See http://ivyspring.com/terms for full terms and conditions.

Received: 2020.05.25; Accepted: 2021.03.15; Published: 2021.03.31

\begin{abstract}
Alternative splicing (AS), as an effective and universal mechanism of transcriptional regulation, is involved in the development and progression of cancer. Therefore, systematic analysis of alternative splicing in pancreatic adenocarcinoma (PAAD) is warranted. The corresponding clinical information of the RNA-Seq data and PAAD cohort was downloaded from the TCGA data portal. Then, a java application, SpliceSeq, was used to evaluate the RNA splicing pattern and calculate the splicing percentage index (PSI). Differentially expressed AS events (DEAS) were identified based on PSI values between PAAD cancer samples and normal samples of adjacent tissues. Kaplan-Meier and Cox regression analyses were used to assess the association between DEAS and patient clinical characteristics. Unsupervised cluster analysis used to reveal four clusters with different survival patterns. At the same time, GEO and TCGA combined with GTEx to verify the differential expression of AS gene and splicing factor. After rigorous filtering, a total of 45,313 AS events were identified, 1,546 of which were differentially expressed AS events. Nineteen DEAS were found to be associated with OS with a five-year overall survival rate of 0.946 . And the subtype clusters results indicate that there are differences in the nature of individual AS that affect clinical outcomes. Results also identified 15 splicing factors associated with the prognosis of PAAD. And the splicing factors ESRPI and RBM5 played an important role in the PAAD-associated AS events. The PAAD-associated AS events, splicing networks, and clusters identified in this study are valuable for deciphering the underlying mechanisms of AS in PAAD and may facilitate the establishment of therapeutic goals for further validation.
\end{abstract}

Key words: genomic analysis, alternative splicing, pancreatic adenocarcinoma, risk model, alternative splicing factors.

\section{Introduction}

Pancreatic adenocarcinoma is one of the most common malignant tumours, ranking fourth in cancer-related deaths, and the incidence of this cancer has increased gradually in recent years $[1,2]$. It is estimated that by 2030, pancreatic adenocarcinoma may exceed colorectal cancer and become the second most common cancer [3]. Given the high mortality rate of pancreatic adenocarcinoma [4], achieving a better understanding of its risk factors is necessary to prevent pancreatic adenocarcinoma.

Currently, alternative splicing (AS) has been the proposed mechanism for tumourigenesis. AS is a key post-transcriptional regulatory mechanism and a major reason for enhancing transcriptome and proteome diversity [5]. Emerging evidence suggests that aberrant alternative splicing is a common event in cancer development and progression and is closely 
related to such processes as proliferation, metastasis, and therapeutic resistance [6-8]. Changes in the expression of key splicing factors can also result in changes in the AS of the target gene. For example, SRSF1 can direct the MYO1B gene to produce AS, which increases the tumourigenic potential of glioma cells via the PDK1 / AKT and PAK / LIMK pathways [9]. CHERP and SR140 regulate the expression of UPF3A splices in CRC by forming protein complexes [10]. In addition, the relevant alternative splicing event can even occur at the lncRNA level, and the lncRNA-PXN-AS1 splicing factor MBNL3 can produce different alternative splicing variants IncRNA-PXN-AS1-L and lncRNA-PXN- AS1-S, which bind to different parts of PXN mRNA, regulate PXN from two different directions and exert different biological functions [11].

Due to the high complexity and heterogeneity of pancreatic adenocarcinoma, the molecular mechanisms of its progression and early metastasis remain unclear $[12,13]$. Alternative splicing is one of the mechanisms leading to the complexity and effectiveness of disease progression. Therefore, it is particularly necessary to identify the link between aberrant alternative splicing and pancreatic adenocarcinoma. Currently, cancer-specific AS events are identified by comparing cancerous tissue with normal control tissue. Systematic profiling of prognostic AS features has been reported in lung [14], breast [15], rectal [16], and thyroid cancers [17]. Currently, there is a lack of comprehensive research examining survival-related AS events in pancreatic adenocarcinoma.

In this study, we compared differentially spliced AS transcripts of pancreatic adenocarcinoma tissues and non-tumour tissues and genome-wide survival-related AS events in 171 patients with pancreatic adenocarcinoma. The RNA-Seq data and the corresponding clinical information of the PAAD cohort were analysed by DEAS and its splicing network by bioinformatics methods. A total of 45,313 AS events were identified, 1546 of which were differentially expressed AS events (DEAS). Nineteen DEAS events were found to be associated with OS with a five-year overall survival rate of 0.946 . The regulatory relationship can be further predicted in the network constructed for the identified prognostic-related splicing factors and alternative splicing events. And the splicing factors ESRP1 and RBM5 played an important role in the PAAD-associated AS events. The PAAD-associated AS events and splicing networks we identified are of great value in deciphering the underlying mechanisms of AS in PAAD and may facilitate the establishment of therapeutic goals for further validation.

\section{Methods}

\section{Alternative Splicing Event Curation from TCGA RNA-seq Data}

The TCGA Data Portal (https://portal.gdc. cancer.gov/projects) provides RNA-seq data for TCGA pancreatic adenocarcinoma. This study used the SpliceSeq tool to analyse AS profiles and assess the splicing patterns of mRNA in pancreatic adenocarcinoma patients. Percent splicing index (PSI), ranging from 0 to 1 , are used to quantify AS events and calculate seven types of alternative splicing events: Exon Skip (ES), Mutually Exclusive Exons (ME), Retained Intron (RI), Alternate Promoter (AP), Alternate Terminator (AT), Alternate Donor site (AD), and Alternate Acceptor site (AA) (Figure 1A).

\section{Differentially Spliced AS Events Analysis}

This study compared the analysed AS spectrum of pancreatic adenocarcinoma samples with normal adjacent cancer samples, identified differential alternative splicing events, and defined p-values less than $0.05, \mathrm{FC}>1.5$ or $\mathrm{FC}<2 / 3$ as differential alternative splicing events. At the same time, the gene expression profiles of pancreatic adenocarcinoma samples and normal adjacent cancer samples were also analysed for differential expression, and differentially expressed genes were identified for further comparison.

\section{Alternative Splicing Events Statistics and GO and KEGG Enrichment Analysis}

This study conducted a comprehensive statistical analysis of alternative splicing events. Traditionally, most studies use Venn diagrams to represent relationships between interactive sets, but alternative splicing events are divided into seven types. We use a more intuitive UpSet diagram for the overall display of alternative splicing events. At the same time, we use clusterProfiler to perform GO function enrichment analysis on survival-related alternative splicing events, to determine important related biological processes, cellular components and molecular functions, and use kobas database for KEGG analysis.

\section{Survival Analysis}

This study included patients with pancreatic adenocarcinoma with complete clinical parameters and at least 90 days of overall survival (OS). For the PSI value of the AS event for each parameter, the patients were divided into two groups by median. Univariate Cox regression was used to identify 
associations between alternative splicing events and OS in each type, with p-values less than 0.05 being defined as survival-related alternative splicing events.

To remove any genes that might not be independent factors in prognostic predictors, multivariate Cox regression was applied to facilitate analysis of survival-related alternative splicing events in seven types. The prognostic risk score is determined by multiplying the linear combination of AS PSI by the corresponding regression coefficient (b) representing the associated weight. The regression coefficients were calculated from a multivariate Cox proportional hazards regression model. The risk score formula is as follows:

$$
\begin{gathered}
\text { Risk Score }=\text { PSI of } A S_{1} \times b_{A S 1}+\text { PSI of } A S_{2} \times b_{A S 2}+\cdots \\
+ \text { PSI of } A S_{n} \times b_{A S n}
\end{gathered}
$$

Finally, seven different types of candidate independent prognostic AS events were combined to construct a final prognostic predictor. In addition, the Kaplan-Meier curve of prognostic factors for pancreatic adenocarcinoma patients was compared within 5 years of OS. The chi-square test was used to compare the difference in survival status between the high-risk group and the low-risk group and to plot the ROC curve to predict the efficiency of each predictive model.

\section{Identify Clusters Associated with Prognosis and Molecular Subtypes}

Alternative splicing events occur very differently at the individual level. To obtain robust classification, we used the unsupervised consensus approach implemented by Consussus Cluster Plus (R package) to identify molecular subtypes of pancreatic adenocarcinoma. Principal component analysis (PCA) were used to assess the optimal $\mathrm{K}$. For the identified molecular subtypes, we start from the survival time to analyse the survival, identify the relationship between subtypes and survival, and further explore the relevant clinical information, trying to find other relationships between clinical information and molecular subtypes.

\section{Combined analysis of differential expression of prognostic factors and splicing factors}

The GEO database verified the differential expression of 18 genes and 15 splicing factors, and 5 datasets (GSE22780, GSE27890, GSE32676, GSE16515, GSE15471) were selected for further analysis. Considering the limited number of normal tissues in the TCGA-PAAD database, we used the Genotype Tissue Expression Database (GTEx) combined with the TCGA database to verify the differential expression of 18 prognostic genes and 15 splicing factors. The GTEx database contains normal tissue samples from 54 human body parts, provides a data resource analysing and visualizing genomics data, thereby enabling researchers to explore and compare genetic alterations across samples.

\section{Splicing Correlation Network Construction}

A list of 67 human splicing factors was created through hand-planned literature and database filtering [18]. Expression of the splicing factor gene in the mRNA splicing pathway was derived from grade 3 mRNA-seq data in TCGA. Survival-related splicing factors were identified by one-way Cox regression analysis $(\mathrm{p}<0.05)$, and correlations between survival-related splicing factor gene expression and survival-related alternative spliced PSI values were analysed by Spearman's test. A choice of p-value less than 0.05 is defined as a significant correlation. The final interaction network between alternative splicing events and splicing factors was constructed using Cytoscape (3.6.0). At the same time, ClueGO (Cytoscape plug-in) was used for GO functional enrichment analysis and KEGG and Reactome pathway enrichment analysis to find significantly related GO terms or pathways. Gene set enrichment analysis (GSEA) in TCGA-PAAD data was performed using GSEA v3 software to evaluate the function of splicing factors ESPR1 and RBM5.

\section{IHC of ESRPI and RBM5 thirty pairs of PC and adjacent normal tissues}

PC and adjacent normal tissues were collected from the First Affiliated Hospital of Soochow University during the period of 2016 to 2019. The experiments were approved by the Ethical Committee of the First Affiliated Hospital of Soochow University and written informed consent was signed by each participant. All samples were incubated using rabbit polyclonal anti-ESRP1 antibody (catalog no. ab262886) and anti-RBM5 antibody (catalog no. AP70787) overnight at $4^{\circ} \mathrm{C}$. The ESRP1 and RBM5 staining index were classified into four groups: score 0 (no staining), score 1 ( $0-20 \%$ of tumor cells stained), score $2(20-50 \%$ of tumor cells stained) and score 3 ( $>50 \%$ of tumor cells stained). We defined that score $>$ 1 indicated tumours with high ESRP1 (or RBM5) expression and score $\leq 1$ indicated low/negative ESRP1 (or RBM5) expression [19].

\section{Statistical Analysis}

All statistical analyses were performed using $\mathrm{R} /$ Bioconductor (version 3.5.1), reported $\mathrm{p}$ values < 0.05 were considered to be statistically significant, and p-values were bilateral. 


\section{Results}

\section{Integrated AS Events in Pancreatic Adenocarcinoma}

In the 171 patients with pancreatic adenocarcinoma (Supplementary Table S1), 45,313 AS events associated with 10,622 genes were found. In detail, we detected 3,657 AAs out of 2,594 genes, 3,118 ADs in 2,210 genes, and 9,325 APs out of 3,724 genes, 8,733 ATs in 3,816 genes, 17,402 ESs in 6,749 genes, 205 MEs in 202 genes, and 2,872 RIs in 1,922 genes (Figure 1B). These results also indicate that a gene may have several types of mRNA splicing events, and one gene may exhibit up to five or even six to seven alternative splicing types, while ES is the dominant type because more than $1 / 3$ of the AS type is an ES event.

\section{Differentially Spliced AS Events in Pancreatic Adenocarcinoma}

Differential splicing analysis showed that 173 alternative splicing events and corresponding 160 genes were defined as up-regulated alternative splicing events in pancreatic adenocarcinoma, 1,373 alternative splicing events and corresponding 1,152 genes were downward adjustments (Figure $1 \mathrm{C}$ and D). In the differential expression analysis, 712 genes were defined as up-regulated differentially expressed genes in pancreatic adenocarcinoma, and 890 genes were defined as down-regulated differentially expressed genes. We compared the genes involved in the differentially alternative splicing event with the differentially expressed genes and found that the genes identified by the two groups have similarities but more obvious differences. Therefore, we believe that the analysis of the alternative splicing event can compensate for the deficiencies of differential expression analysis (Figure 1D).
A

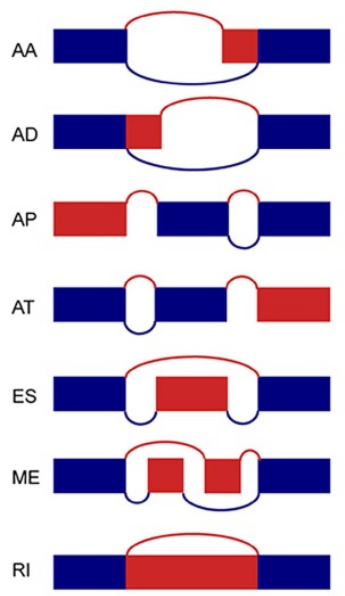

B

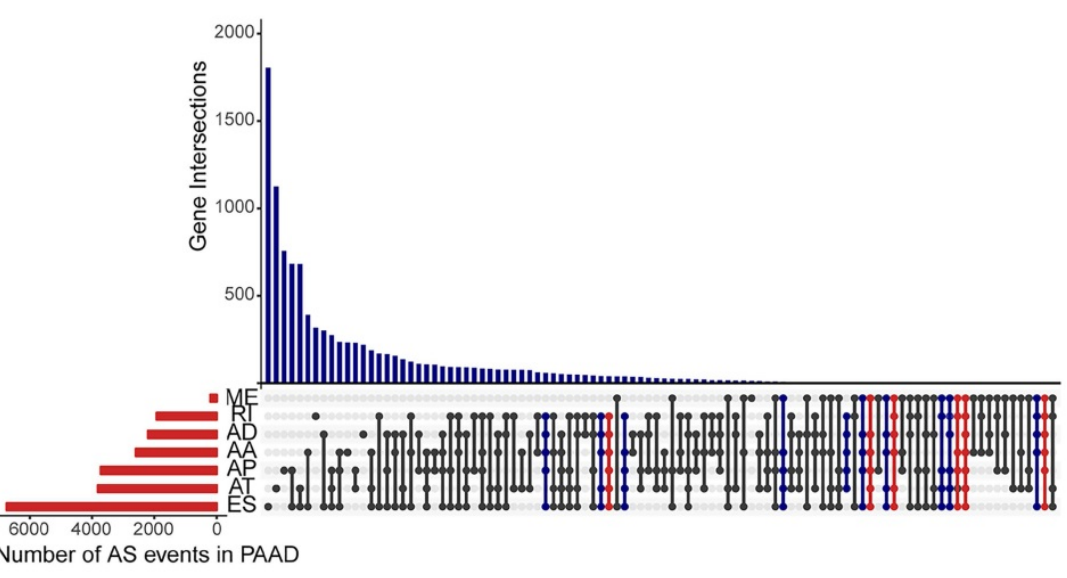

C

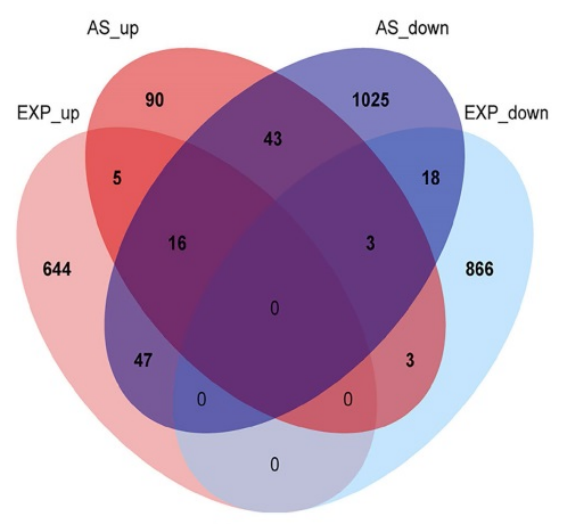

D



Figure 1. Overview of seven types of alternative splicing and differentially spliced AS events analysis in pancreatic adenocarcinoma. (A) Schematic diagram of the alternative splicing type. (B) Number of alternative splicing events of each type and distribution of genes involved in alternative splicing events. Five alternative splicing types are marked in blue, reaching 6-7 alternative splicing types marked in red. (C) Differentially alternative splicing events involve Venn plots of genes and differentially expressed genes, showing the number of their isomorphisms. (D) Volcanic maps of differentially alternative splicing events, where blue represents a downregulated differential alternative splicing event, red represents an upregulated alternative splicing event, and grey represents a non-differential alternative splicing event. 


\section{Survival Associated Alternative Splicing Events in Pancreatic Adenocarcinoma}

Before investigating the prognostic value of the mRNA splicing event, a univariate survival test was performed to assess the relationship between clinical parameters and outcomes in TCGA pancreatic adenocarcinoma. In the pancreatic adenocarcinoma cohort, TNM staging (HR = 1.457, 95\% CI: 1.064-1.994, $\mathrm{P}=0.0189)$ and grade classification $(\mathrm{HR}=1.421,95 \%$ CI: $1.071-1.885, \quad \mathrm{P}=0.0148$ ) were significantly associated with OS. The results of this preliminary assessment indicate that the survival data of pancreatic adenocarcinoma samples in TCGA, although containing censored data, is informative and suitable for further molecular studies. To investigate the prognostic value of AS events in patients with pancreatic adenocarcinoma, we used univariate Cox regression analysis to assess the prognostic impact of differential alternative splicing events on patients with pancreatic adenocarcinoma. We detected a total of 130 survival-related alternative splicing events $(p<$ 0.05 ) in differentially alternative splicing events. The Circos plot shows the details of survival-related AS events and their associated genes (Figure 2A) and takes six alternative splicing types (alternative splicing events associated with survival are not identified in the ME type). The most important survival-related alternative splicing events of TOP5 are plotted in the forest map (Figure 2B). Subsequently, GO functional enrichment analysis and KEGG analysis were performed on 116 genes involved in survival-related alternative splicing events $(p<0.05)$. The prognostic factors were enriched in cellular functions, such as RNA splicing, RNA splicing regulation, mRNA processing regulation, and the mitochondrial inner membrane, and enriched in metabolic pathway and spliceosome in KEGG pathway (Figure 2C).

\section{Prognostic Predictors for Pancreatic Adenocarcinoma}

To detect independent prognostic factors in patients with pancreatic adenocarcinoma, we selected survival-related alternative splicing events $(\mathrm{p}<0.01)$ as candidates and multivariate Cox regression analysis to identify independent prognostic factors in the six alternative splicing types retained $(\mathrm{p}<0.05)$. Two independent prognostic factors associated with $\mathrm{AD}$ were obtained: three independent prognostic factors associated with $\mathrm{AP}$, nine independent prognostic factors associated with $\mathrm{AT}$, three independent prognostic factors associated with ES, and two independent prognostic factors associated with RI. Independent prognostic factors associated with AA were not identified (Table 1). Five different types of independent prognostic alternative splicing events were further combined to construct a final prognostic predictor. In our data analysis of each type of splicing pattern, the use of different types of alternative splicing events to construct a prognostic model demonstrated a significant ability to predict outcomes in patients with pancreatic adenocarcinoma, and these AS features may be a reliable predictor of prognosis in patients with pancreatic adenocarcinoma (Figure 3A-E, Supplementary Figure S1A-E). In particular, a prognostic model constructed from a single AP model showed better performance predicted by the five prognostic models $(\mathrm{ROC}=0.824)$ (Figure 3G).

In addition, five different types of candidate independent prognostic AS events (19 total splicing events) were combined to construct a final prognostic predictor. It is worth noting that the final prognostic model showed better performance than each single type of splicing pattern in predicting $(\mathrm{ROC}=0.946)$, as shown in Figure 3F (Supplementary Figure S1F) and G. It is conceivable that the final combined prognostic model is more efficient than other prognostic models. At the same time, we compare the ROC curves of the 3,5 and 7 years of the final prognostic model. The model exhibited good predictive power (Figure $3 \mathrm{H}$ ).

Table 1. Independent prognostic factors in alternative splicing types with pancreatic cancer $(p<0.05)$.

\begin{tabular}{lllllll}
\hline AS_ID & Gene & \multicolumn{2}{l}{ Type } & P-value & HR & Low 95\%CI High 95\%CI \\
\hline ID_89320 & LAS1L & AD & 0.0031 & 0.0001123 & $2.71 \mathrm{E}-07$ & 0.0464984 \\
ID_42666 & SUPT4H1 & AD & 0.00821 & $1.23 \mathrm{E}-05$ & $2.80 \mathrm{E}-09$ & 0.0537494 \\
ID_9575 & FAM72A & AP & 0.00014 & 0.0771458 & 0.0206222 & 0.2885959 \\
ID_22286 & WIBG & AP & 0.0812 & 60.139324 & 0.6017212 & 6010.6543 \\
ID_48878 & PDCD5 & AP & 0.89371 & 0.8453955 & 0.0719646 & 9.9311824 \\
ID_85365 & LYNX1 & AT & $4.73 E-05$ & 0.2493761 & 0.1277371 & 0.4868471 \\
ID_15925 & C11orf31 & AT & 0.00039 & $3.80 \mathrm{E}-21$ & $1.94 \mathrm{E}-32$ & $7.45 \mathrm{E}-10$ \\
ID_32153 & TMED3 & AT & 0.00165 & $1.28 \mathrm{E}+267$ & $5.91 \mathrm{E}+100$ & Inf \\
ID_9577 & FAM72A & AT & 0.00549 & 0.0860268 & 0.015227 & 0.4860172 \\
ID_63032 & EDEM1 & AT & 0.00859 & $3.673 \mathrm{E}+09$ & 270.12343 & $4.995 \mathrm{E}+16$ \\
ID_2074 & PPCS & AT & 0.02444 & $4.34 \mathrm{E}-12$ & $5.50 \mathrm{E}-22$ & 0.0342591 \\
ID_78951 & KLHL7 & AT & 0.03529 & $3.99 \mathrm{E}-13$ & $1.14 \mathrm{E}-24$ & 0.1398364 \\
ID_86327 & CLTA & AT & 0.04937 & $5.365 \mathrm{E}+14$ & 1.0987634 & $2.62 \mathrm{E}+29$ \\
ID_60044 & PSMA7 & AT & 0.11692 & 213902157 & 0.0082526 & $5.544 \mathrm{E}+18$ \\
ID_67027 & MRPS22 & ES & 0.00097 & $1.52 \mathrm{E}-09$ & $8.76 \mathrm{E}-15$ & 0.0002621 \\
ID_680 & AGTRAP & ES & 0.00116 & $1.436 \mathrm{E}+11$ & 26774.479 & $7.703 \mathrm{E}+17$ \\
ID_41127 & VPS25 & ES & 0.0022 & $1.33 \mathrm{E}-35$ & $6.37 \mathrm{E}-58$ & $2.79 \mathrm{E}-13$ \\
ID_16163 & CYB561A3 & RI & 0.00051 & 0.000185 & $1.45 \mathrm{E}-06$ & 0.0235353 \\
ID_61140 & RANBP1 & RI & 0.2219 & 0.0348577 & 0.0001597 & 7.6079761 \\
\hline
\end{tabular}

\section{Prognosis-Associated Molecular Subtype Cluster}

We further identified different AS patterns by unsupervised analysis of all samples based on 19 alternative splicing events associated with prognosis. By combining the Elbow method to determine the optimal number of clusters (Figure 4A), the PCA showed a relatively stable partitioning of the samples in the 4 clusters (Figure 4B), we finally determined 
four sets of samples as follows: $\mathrm{C} 1(\mathrm{n}=35,21.0 \%), \mathrm{C} 2$ $(\mathrm{n}=27,16.2 \%), \mathrm{C} 3(\mathrm{n}=50,29.9 \%)$, and C4 $(\mathrm{n}=55$, $32.9 \%$ (Figure 4C).We then performed a Kaplan-Meier analysis to assess the relationship between clustering and prognosis. The results indicate that clusters are associated with different survival patterns, with Cluster3 correlating with poor outcomes in survival analysis (Figure 4D). At the same time, we further analysed the relevant clinical information and found that some related information was not randomly distributed, such as survival time (OS $>5$ years or $<5$ years), survival status (Alive or Dead), and stage in four clusters. There was a significant difference in the histological type (chi-square test, $\mathrm{p}<0.05$ ) (Figure $4 \mathrm{E}$ ). Therefore, we can also identify molecular subtype clusters associated with prognosis through alternative splicing events, and the results of the study indicate that there are differences in the nature of individual AS that affect clinical outcomes.
A

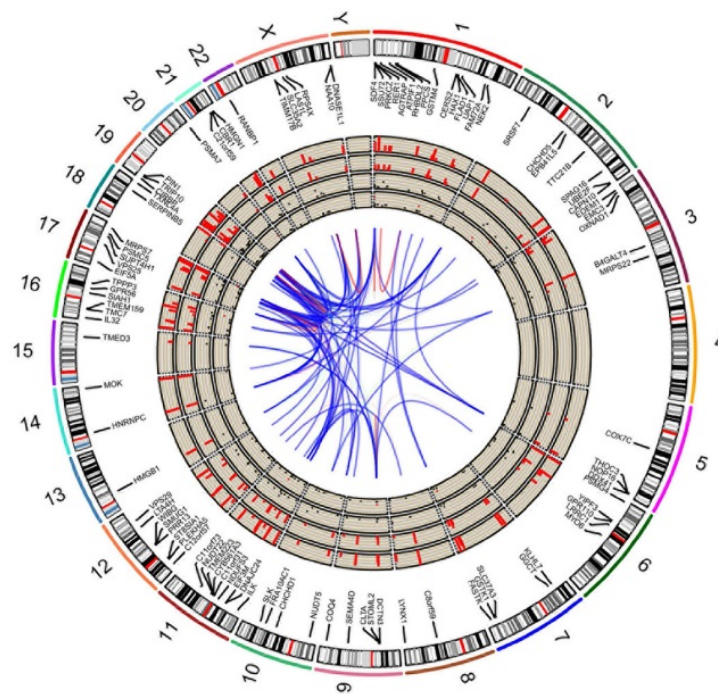

C



B
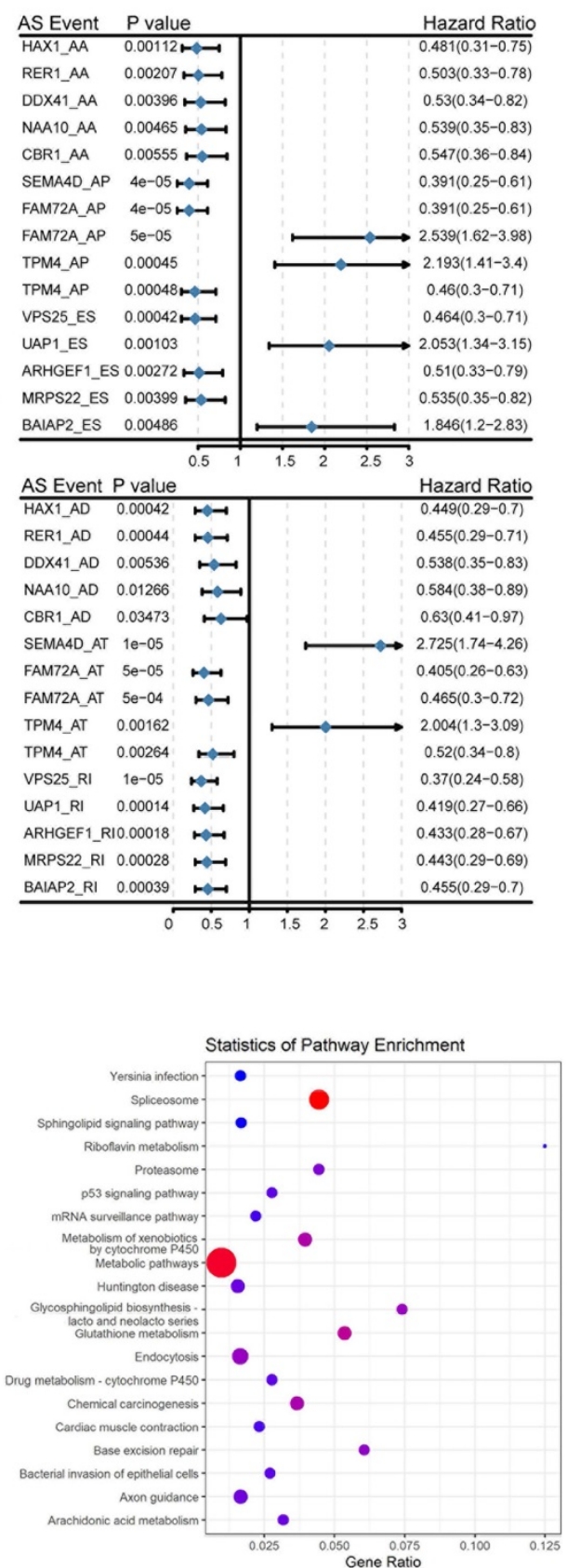

Figure 2. Alternative splicing events statistics and GO functional enrichment analysis. (A) The Circos diagram of the survival-related alternative splicing events and its related genes, the Circos panel from the outside to the inside, is expressed as follows: chromosome number, genomic axis, survival-related alternative splicing event-related genes, names of related genes, the number of related genes occurring in the overall alternative splicing events $(1-10$ (>10), and showing $1-10$ different heights, over 10 calculations on time), the number of alternative splicing types of related genes in the overall events, the $p$ value of the relevant gene in the difference analysis (expressed by the conversion value of - $\log 10$ ( $\mathrm{p}$-values), and the higher the height, the more significant the $\mathrm{p}$ value), the fold change value of the relevant gene in the difference analysis (where red represents upregulation and black represents downregulation), correlation between genes. (B) A forest map of the most important TOP5 survival-related alternative splicing events in each of the alternative splicing types after single factor Cox regression analysis. (C) GO and KEGG Enrichment analysis results of survival-related alternative splicing event-related genes after one-way Cox regression analysis. 
A


E

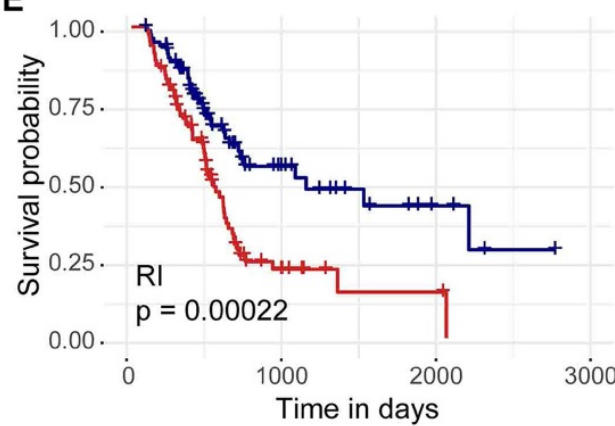

B



D

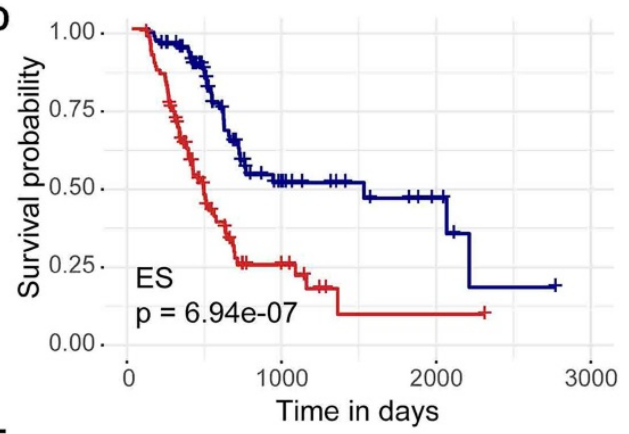

F

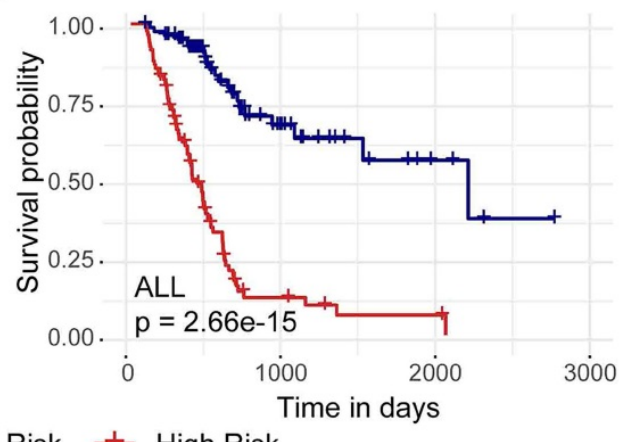

Group — Low Risk — High Risk
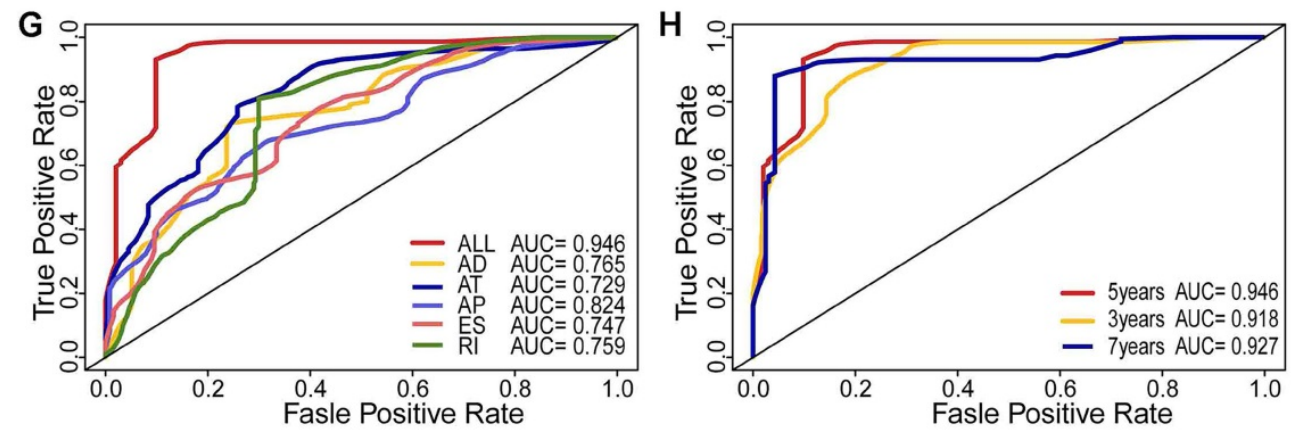

Figure 3. Kaplan-Meier plots and ROC curves of predictive Kaplan-Meier plots and ROC curves of predictive factors in the TCGA pancreatic adenocarcinoma cohort. (A)-(E) Kaplan-Meier curves plotted for prognostic models of each type of alternative splicing event. (F) Kaplan-Meier curves drawn from the prognostic model after integration of each type. The red line represents the high-risk group, and the blue line represents the low-risk group. (G) ROC curves for each type and post-integration alternative splicing event. (H) 3, 5, and 7 year ROC curves for alternative splicing events after integration.

\section{Combined analysis of differential expression of AS-associated genes}

In addition, in order to further identify the reliability of the prognostic factors, other databases besides TCGA were used to perform. Although the GEO and GTEx databases do not contain RNA-Seq information, they are of great significance for verifying the differential expression of prognostic factors. Five GEO datasets and TCGA combined GTEx datasets were used to verify the differential expression of prognostic factors. As shown in Table 2, most of the results show that these prognostic factors have expression differences in GEO and TCGA combined GTEx. 
Table 2. The 18 genes in alternative splicing types differentially expressed with pancreatic cancer.

\begin{tabular}{|c|c|c|c|c|c|c|}
\hline \multirow[t]{2}{*}{ Gene } & \multicolumn{3}{|c|}{ Five GEO datasets } & \multicolumn{3}{|c|}{ TCGA combined GTEx datasets } \\
\hline & $\log _{2} \mathrm{FC}$ & $P$ value & Adjusted $p$ value & $\log _{2} \mathrm{FC}$ & $P$ value & Adjusted $p$ value \\
\hline AGTRAP & 28.41 & 0.060065 & 0.120577 & 72921.16 & 6.87E-07 & 8.83E-07 \\
\hline C11orf31 & 382.54 & 0.052535 & 0.120577 & 41386.45 & $3.55 \mathrm{E}-13$ & 6.39E-13 \\
\hline CLTA & 248.16 & 0.023342 & 0.120577 & 156514.22 & $2.58 \mathrm{E}-20$ & $5.16 \mathrm{E}-20$ \\
\hline CYB561A3 & 203.78 & 0.042529 & 0.120577 & -52884.05 & $3.10 \mathrm{E}-49$ & $6.97 \mathrm{E}-49$ \\
\hline EDEM1 & 103.37 & 0.025767 & 0.120577 & -324291.22 & 7.27E-69 & $1.87 \mathrm{E}-68$ \\
\hline FAM72A & 23.31 & 0.026911 & 0.120577 & -130.57 & 0.639447 & 0.677062 \\
\hline KLHL7 & 39.13 & 0.080385 & 0.120577 & 6569.78 & 7.97E-10 & 1.19E-09 \\
\hline LAS1L & 17.24 & 0.263855 & 0.279376 & -98400.06 & 2.42E-109 & 8.71E-109 \\
\hline LYNX1 & 23.06 & 0.125112 & 0.150135 & 366.73 & 0.9522 & 0.9522 \\
\hline MRPS22 & 129.97 & 0.12067 & 0.150135 & -6471.30 & 0.000548 & 0.000617 \\
\hline PDCD5 & 6.84 & 0.656805 & 0.656805 & 24458.05 & $5.70 \mathrm{E}-08$ & 7.90E-08 \\
\hline PPCS & 240.21 & 0.054586 & 0.120577 & 26130.70 & $1.81 \mathrm{E}-06$ & 2.17E-06 \\
\hline PSMA7 & 344.63 & 0.017642 & 0.120577 & 682454.68 & $6.52 \mathrm{E}-174$ & 5.87E-173 \\
\hline RANBP1 & 26.76 & 0.071818 & 0.120577 & 225448.40 & $2.50 \mathrm{E}-142$ & $1.50 \mathrm{E}-141$ \\
\hline SUPT4H1 & 82.57 & 0.076974 & 0.120577 & -912175.00 & 2.29E-200 & 4.13E-119 \\
\hline TMED3 & 46.29 & 0.122238 & 0.150135 & -64975.98 & $2.15 \mathrm{E}-11$ & $3.52 \mathrm{E}-11$ \\
\hline VPS25 & 50.40 & 0.06168 & 0.120577 & 115857.38 & $4.38 \mathrm{E}-87$ & $1.31 \mathrm{E}-86$ \\
\hline WIBG & 30.67 & 0.184132 & 0.207148 & 107219.64 & $1.35 \mathrm{E}-125$ & 6.07E-125 \\
\hline
\end{tabular}

A

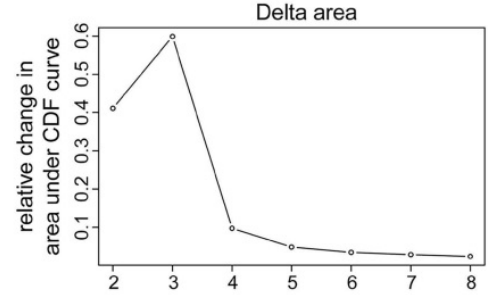

C

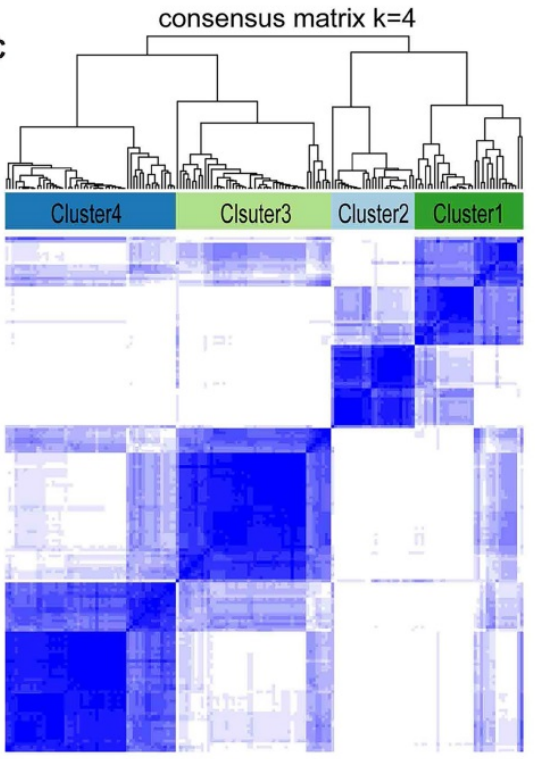

B
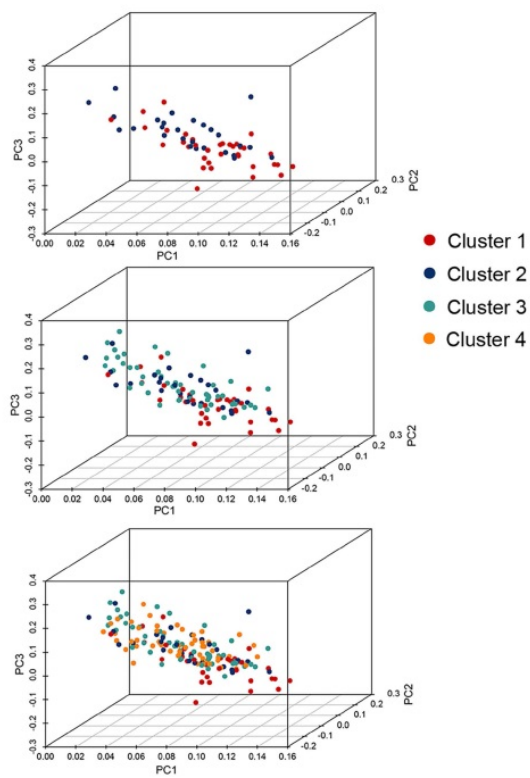

D

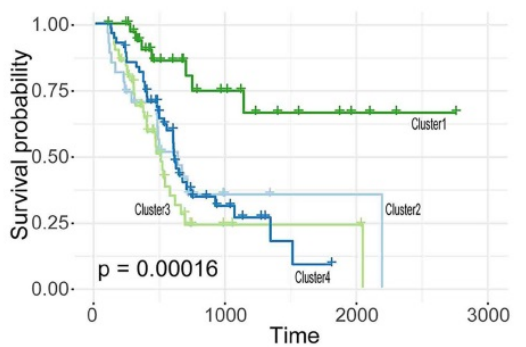



Figure 4. Prognosis-associated molecular subtype cluster analysis. (A)-(B) Statistical analysis of Elbow for different numbers of clusters $(k=2$ to 8$)$ and PCA analysis for $K=4$. (C) The consensus matrix heat map defines four sample clusters with consensus values ranging from 0 (white, samples never gathered together) to 1 (dark blue, samples are always clustered together). (D) Survival analysis in the identified four sample clusters. (E) The distribution of each clinical information in four sample clusters. 
Table 3. The 15 splicing factors differentially expressed in pancreatic cancer.

\begin{tabular}{|c|c|c|c|c|c|c|}
\hline \multirow[t]{2}{*}{ Gene } & \multicolumn{3}{|c|}{ Five GEO datasets } & \multicolumn{3}{|c|}{ TCGA combined GTEx datasets } \\
\hline & $\log _{2} \mathrm{FC}$ & $P$ value & Adjusted $p$ value & $\log _{2} \mathrm{FC}$ & $P$ value & Adjusted $p$ value \\
\hline ELAVL2 & 7.75 & $6.38 \mathrm{E}-12$ & $7.98 \mathrm{E}-12$ & 619.73 & 1.93E-05 & 2.41E-05 \\
\hline ELAVL4 & -65.99 & 4.09E-35 & 3.07E-34 & 2675.26 & 0.003551 & 0.004098 \\
\hline ESRP1 & 407.94 & $1.29 \mathrm{E}-25$ & $3.86 \mathrm{E}-25$ & 73705.38 & $6.95 \mathrm{E}-17$ & $1.16 \mathrm{E}-16$ \\
\hline HNRNPAO & 186.84 & $1.12 \mathrm{E}-14$ & $1.53 \mathrm{E}-14$ & 68273.36 & $4.83 \mathrm{E}-42$ & $1.03 \mathrm{E}-41$ \\
\hline HNRNPL & -59.93 & $9.84 \mathrm{E}-12$ & 1.14E-11 & 43150.39 & 2.33E-17 & $4.36 \mathrm{E}-17$ \\
\hline HNRNPM & 12.71 & 0.099515 & 0.099515 & 111358.08 & 2.73E-61 & $6.83 \mathrm{E}-61$ \\
\hline HNRNPU & -98.38 & 2.23E-07 & 2.39E-07 & 19590.14 & 0.353961 & 0.379244 \\
\hline NOVA1 & 28.82 & 8.53E-26 & $3.20 \mathrm{E}-25$ & 388.42 & 0.654919 & 0.654919 \\
\hline RBM4 & 69.88 & 4.53E-26 & $2.26 \mathrm{E}-25$ & 98766.58 & $1.70 \mathrm{E}-86$ & 5.09E-86 \\
\hline RBM5 & -414.03 & 5.09E-37 & 7.63E-36 & -77494.35 & $1.25 \mathrm{E}-86$ & 4.67E-86 \\
\hline$R B M X$ & 30.34 & $4.02 \mathrm{E}-23$ & $1.01 \mathrm{E}-22$ & -171121.44 & $1.92 \mathrm{E}-100$ & $1.44 \mathrm{E}-99$ \\
\hline SF1 & -89.17 & 7.94E-23 & $1.70 \mathrm{E}-22$ & -91532.75 & 5.38E-15 & 8.07E-15 \\
\hline SFPQ & 330.84 & 2.40E-19 & $4.51 \mathrm{E}-19$ & -181385.34 & $1.94 \mathrm{E}-08$ & $2.65 \mathrm{E}-08$ \\
\hline TRA2A & 113.27 & $1.92 \mathrm{E}-15$ & 2.87E-15 & -1377268.74 & $3.74 \mathrm{E}-88$ & $1.87 \mathrm{E}-87$ \\
\hline$Y B X 1$ & -698.48 & $8.45 \mathrm{E}-18$ & $1.41 \mathrm{E}-17$ & 1480274.26 & $2.32 \mathrm{E}-118$ & 3.49E-117 \\
\hline
\end{tabular}

\section{Network of Survival-Associated AS Splicing Factors}

Through manual planning of literature and database screening, a list of 67 human splicing factors was created to further analyse the expression of splicing factor genes in the mRNA splicing pathway from TCGA. To determine which splicing factors are associated with AS events associated with survival in pancreatic adenocarcinoma, we performed a survival analysis of splicing factors, and the results showed that 15 splicing factors were significantly associated with overall survival (Figure 5A, Supplementary Table S2). The heat map shows the details of the 15 splicing factors in the TCGA cohort (Figure 5B).

Through correlation analysis between the expression values of survival-related alternative splicing factors and the constructed prognostic models, we observed that the expression levels of most splicing factor genes were negatively correlated with the PSI of prognostic signals (Figure 6A). In addition, the Spearman test was used to investigate the correlation between the PSI values of the most important AS events and the expression of survival-related splicing factors. Among these factors, 14 survival-related splicing factors (grey points) were significantly associated with 18 survival-related AS events (corresponding to 17 genes, 16 protective factors (blue dots) and 1 risk factor (red dots)), which constituted 129 line alternative splicing networks with interactions (Figure 5B). Subsequently, ClueGO was used to perform enrichment analysis on the relevant genes in the network, and the significantly enriched GO term and related pathways were found. The ClueGO enrichment results are closely related to the pre-transcriptional regulation of mRNA, such as pre-mRNA binding, mRNA stabilization, alternative mRNA splicing (via spliceosome), mRNA splicing, and formation of the spliceosomal E complex (Figure $5 C)$.
In addition, we also used five GEO datasets and the TCGA combined GTEx dataset to verify the differential expression of splicing factors, and almost all splicing factors have obvious expression differences in the GTEx combination of GEO and TCGA (Table 3).

\section{Specific analysis of splicing factors ESRP I and RBM5}

Since splicing factors ESRP1and RBM5 perform best in survival analysis, we focus on analyzing the respective regulatory relationships between ESRP1 and RBM5 in the SF-AS network. The network revealed that the correlation between the splicing factor ESRP1 and alternative splicing events was 14-fold, and the correlation between the splicing factor RBM5 and alternative splicing events was also 13-fold (Figure 6B). Specifically, in the AT splicing type, the splicing factor ESRP1 is negatively correlated with FAM72A, and the splicing factor RBM5 is positively correlated with C11orf31 (Figure 7A). GESA enrichment analysis results show that ESRP1 and RBM5 are significantly enriched in splice some pathway, while ESRP1 plays an important role in the aminoacyl tRNA biosynthesis pathway, and RBM5 participates in the P53 signaling pathway (Figure 7B). Finally, we plotted the prognostic curve of ESRP1 and RBM5 in TCGA (Figure 7C). And a survival analysis based on ESRP1 or RBM5 signature was further performed in the subgroup of patients with different clinical variables in the TCGA cohort. For the TCGA cohort, after stratifying the clinicopathological characteristics by gender, age, tumor size, pathological stage and AJCC stage, ESRP1 showed significance in age $(<=60)$, male, and AJCC stage I+II (Supplementary Figure S2). RBM5 showed significance in age $(>60)$, male, pathologic stage I+II, AJCC stage II+III+IV and Tumor size T1+T2 (Supplementary Figure S3). 
A

TCGA hazard ratio $p$ value

\begin{tabular}{|c|c|c|c|c|}
\hline ESRP1 & $1.970(1.278-3.036)$ & & $\longmapsto$ & 0.002147233 \\
\hline RBM5 & $0.522(0.337-0.810)$ & $\mathrm{F}-\mathrm{H}$ & & 0.003678339 \\
\hline TRA2A & $0.534(0.344-0.827)$ & $1=-1$ & & 0.004917849 \\
\hline HNRNPL & $1.842(1.201-2.824)$ & & & 0.005102569 \\
\hline HNRNPAO & $0.542(0.350-0.838)$ & $1=-1$ & & 0.00593601 \\
\hline SF 1 & $0.549(0.357-0.845)$ & $1=-1$ & & 0.006348607 \\
\hline HNRNPM & $0.559(0.362-0.862)$ & $1=-1$ & & 0.00844368 \\
\hline ELAVL2 & $0.607(0.396-0.932)$ & $10-1$ & & 0.022389608 \\
\hline RBMX & $0.610(0.399-0.934)$ & & & 0.02294624 \\
\hline RBM4 & $1.621(1.059-2.482)$ & & & 0.02606448 \\
\hline HNRNPU & $1.610(1.043-2.484)$ & & & 0.031507454 \\
\hline SFPQ & $0.637(0.416-0.974)$ & . & & 0.037549984 \\
\hline YBX1 & $1.561(1.024-2.382)$ & & & 0.038640442 \\
\hline NOVA1 & $0.639(0.415-0.985)$ & $1=-1$ & & 0.042670457 \\
\hline ELAVL4 & $0.648(0.424-0.990)$ & $1=-1$ & & 0.044948981 \\
\hline
\end{tabular}

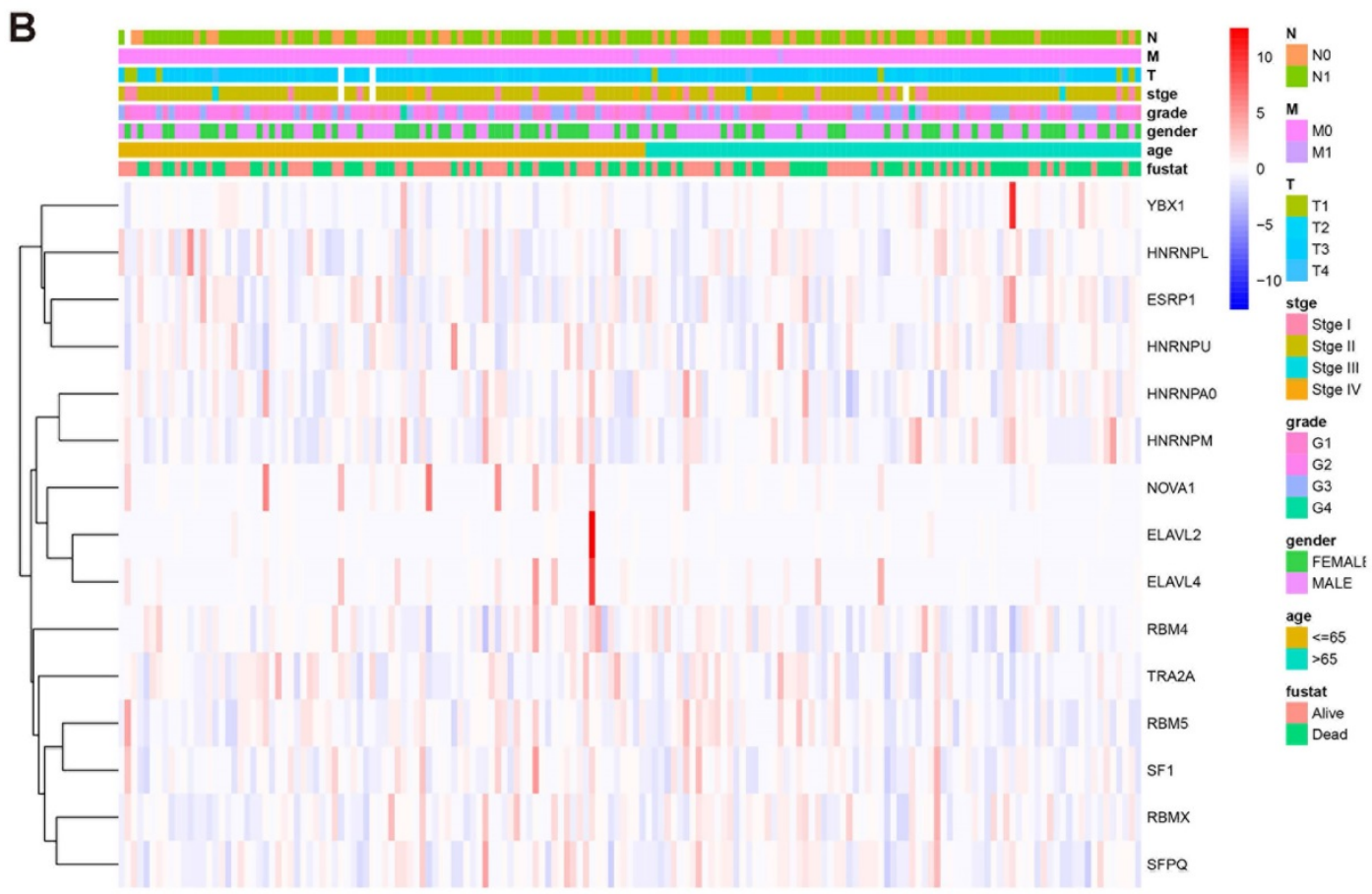

Figure 5. Survival analysis and detail distribution of splicing factors. (A) Forest plots visualizing the $\mathrm{p}$-value of 15 splicing factors identified by survival analysis of TCGA. (B) The details of the 15 splicing factors in the TCGA cohort.

\section{Immunohistochemistry (IHC) of splicing factors ESRPI and RBM5}

Immunostaining images of ESRP1 and RBM5 in thirty pairs of PC and adjacent normal tissues were displayed in Figure 8. ESRP1 and RBM5 were primarily expressed in cytoplasm. The positive staining of ESRP1 was detected in the majority of PC tissues ( $n=30$, positive 97\%) but less frequently in adjacent normal tissues $(n=30$, positive $17 \%)$.
Contrarily, the positive staining of RBM5 was observed weaker in PC tissues ( $\mathrm{n}=30$, positive $20 \%$ ) but stronger in adjacent normal tissues $(n=30$, positive 93\%). IHC proved that ESRP1 was up-regulated in PC compared with adjacent normal tissues, and the RBM5 was down-regulated in PC tissues. The experimental results of immunohistochemistry support the analysis content of splicing factors ESRP1 and RBM5 in the bioinformatics database. 
A
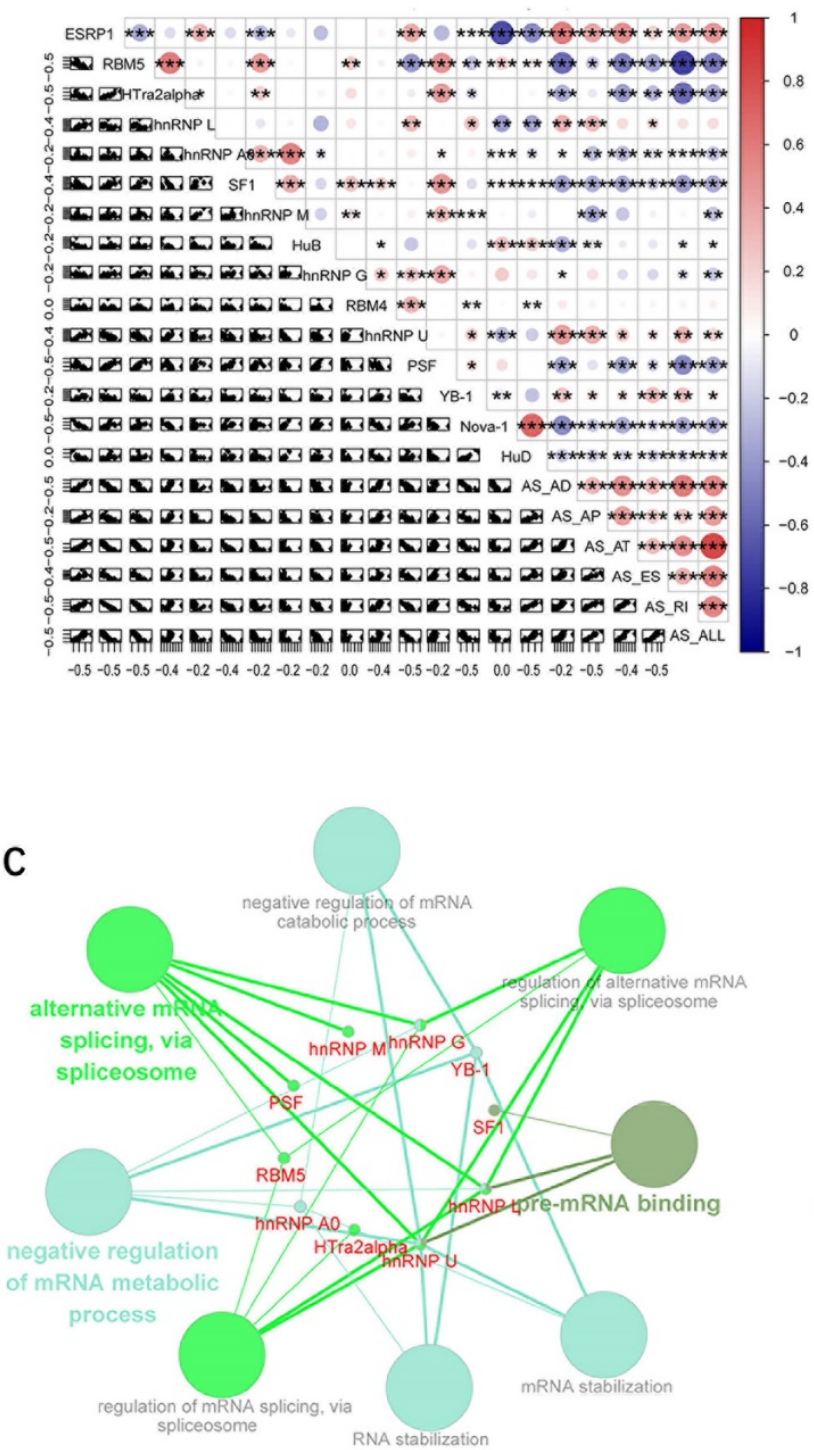

B
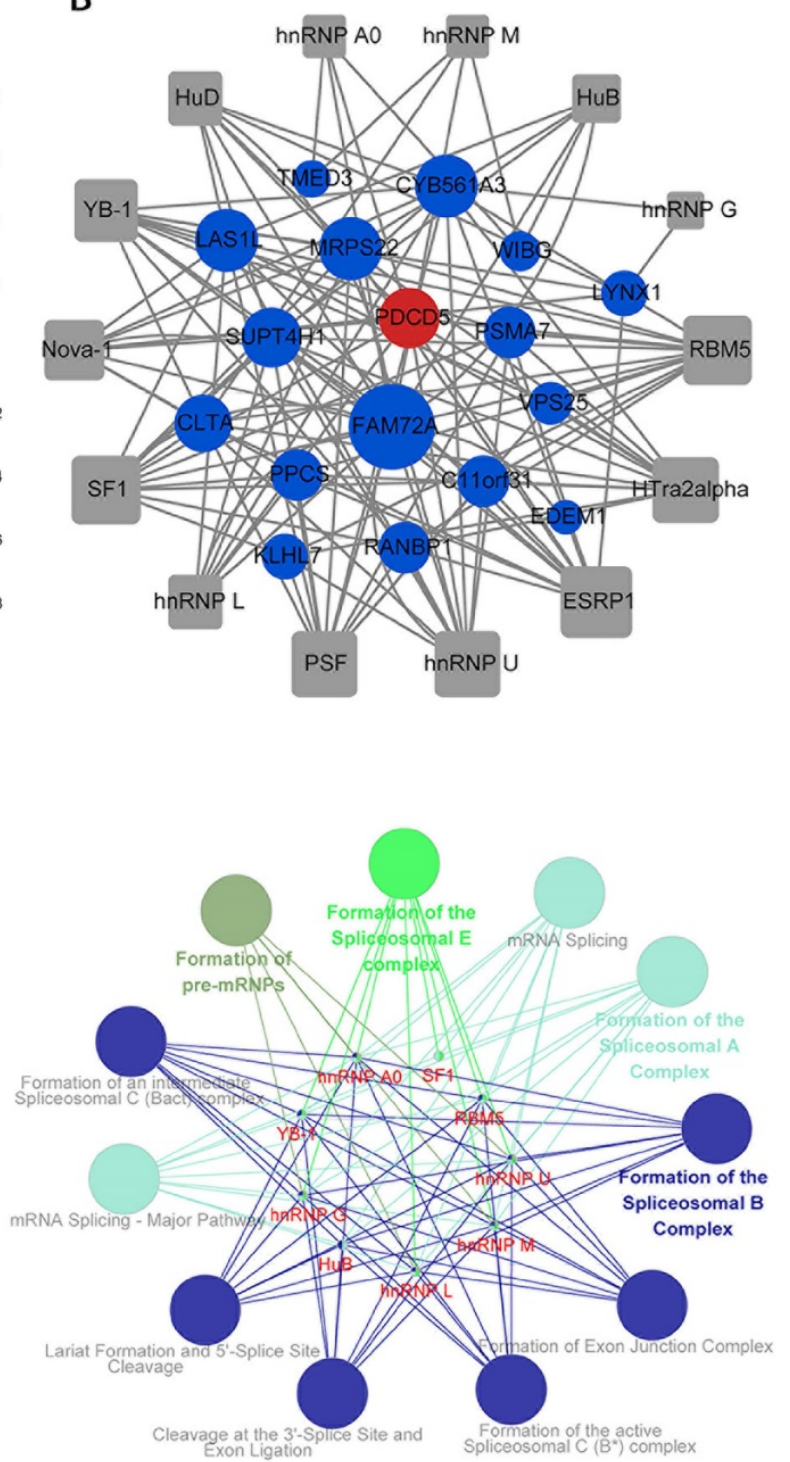

Figure 6. Correlation analysis of splicing factors and prognosis-related AS predictors. (A) Correlation analysis between splicing factors and AS prognostic predictors. The upper panel shows the correlation between correlation coefficient and splicing factor expression and PSI values of prognostic-related AS events. The size and colour of the circle represent the weight of the correlation coefficient, $* p<0.05$, $* * p<0.01$, $* * * p<0.001$, and the scatter plot shows the correlation between the expression of the splicing factor and the PSI value of the survival-related AS event. (B) Alternative splicing network: the square node is the splicing factor, the circular node is the gene involved in the prognosis-related alternative splicing event, the blue node is the protective factor, and the red node is the risk factor. (C) Significantly enriched GO term, significantly enriched KEGG or Reactome pathway.

\section{Discussion}

It has been previously shown that alternative splicing events contribute to cancer development and progression, and all processes of cancer (such as angiogenesis, cell proliferation, invasion, and immune response) are associated with alternative splicing of key genes $[6,7,20]$. For example, the effect of CRKL on alternative splicing may be significantly associated with tumourigenesis in cervical cancer [21]. SRSF3 promotes the expression of splice-like GRa which, in turn, regulates the migration and migration of breast cancer cells by RACK1 [22]. HNRNPLL regulates AS of CD44 to promote proliferation and metastasis of colorectal cancer [23]. Changes in splicing factor expression that regulate alternative splicing events have also been identified in lung cancer, affecting such proteins as QKI, RBM4, RBM5, RBM6, RBM10 and SRSF1, which are important splicing factors in lung cancer alternative splicing events [24]. Even more notable is that similar to the aforementioned IncRNA-PXN-AS1 [11], the isoform due to alternative splicing can have the opposite effect on cancer. For example, in oral squamous cell carcinoma, depending on whether exon 23 is missing, STAT3 produces two variants by alternative splicing. STAT3a encodes a full-length oncogenic STAT3a protein, while STAT3 $\beta$ encodes a tumour-suppressed STAT3 $\beta$ protein. Specifically, PCBP1 is a key splicing factor that regulates alternative splicing of STAT3 exon 23 and 
promotes the transformation from oncogenic STAT3a to tumour suppressor STAT3 $\beta$ [25]. Downregulation of ITSN1-L expression and upregulation of ITSN1-S expression may be one of the mechanisms of glioma

A

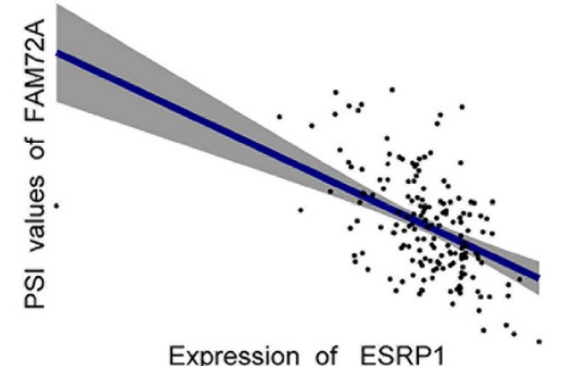

B proliferation and invasion, suggesting that regulation at the level of splicing may be an effective therapeutic strategy [26].

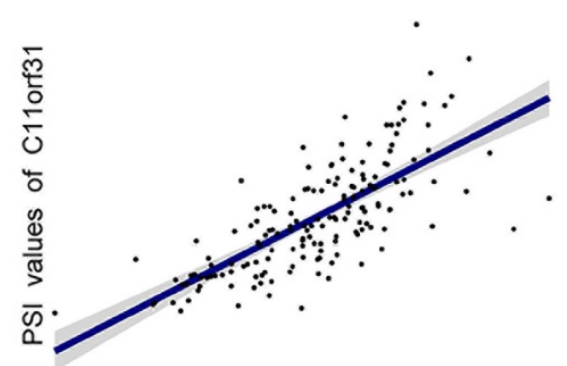

Expression of RBM5

\begin{tabular}{|c|c|}
\hline Name & NES \\
\hline KEGG_SPLICEOSOME & 1.691727 \\
\hline KEGG_P53_SIGNALING_PATHWAY & 1.578314 \\
\hline KEGG_STEROID_BIOSYNTHESIS & 1.573495 \\
\hline KEGG_PENTOSE_PHOSPHATE_PATHWAY & 1.551118 \\
\hline KEGG_ONE_CARBON_POOL_BY_FOLATE & 1.521618 \\
\hline
\end{tabular}

Enrichment plot: KEGG_P53_SIGNALING_PATHWAY KEGG_AMINOACYL_TRNA_BIOSYNTHESIS

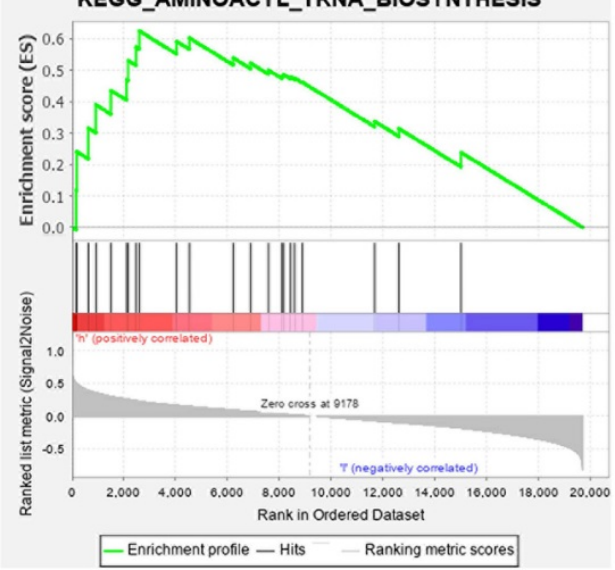

C

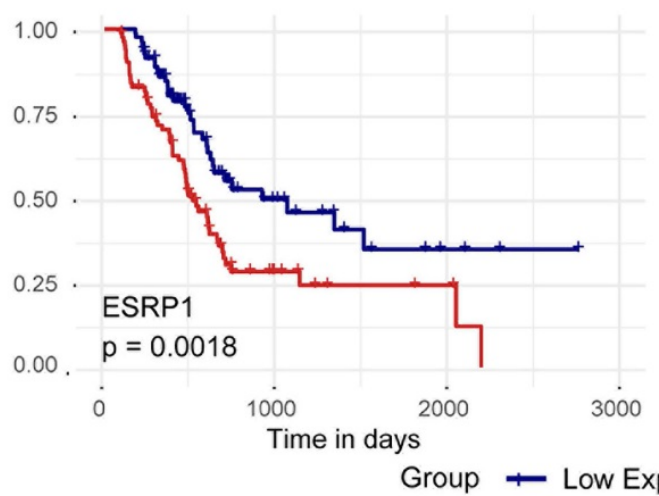

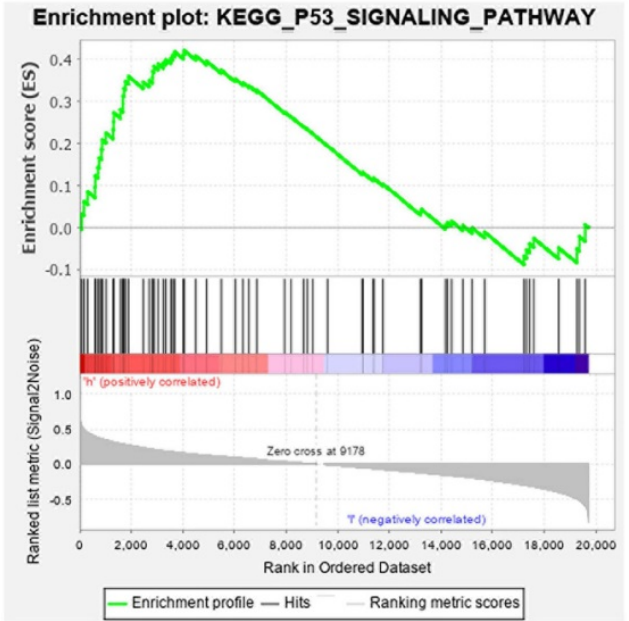

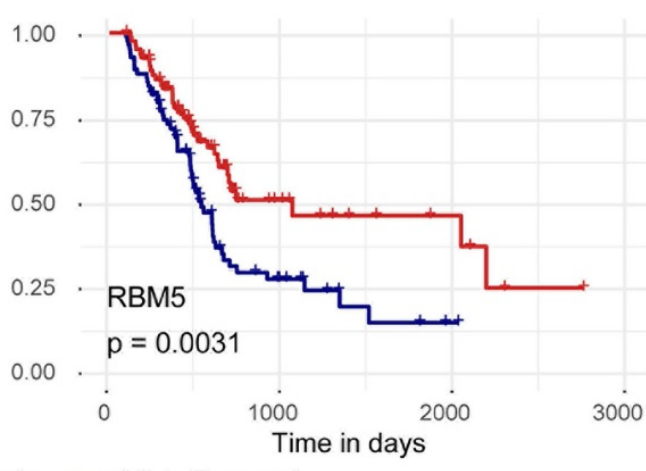

Figure 7. Specific analysis of splicing factors ESRP1 and RBM5. (A) ESRP1 is negatively correlated with FAM72A, and RBM5 is positively correlated with C1 lorf31. (B) GESA analysis of splicing factors ESRPI and RBM5. (C) Survival curves of the identified survival-associated splicing factors ESRPI and RBM5, with the red line representing the high expression group and the blue line representing the low expression group. 
A

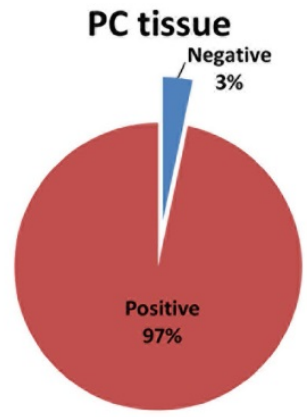

Staining instensity of ESRP1
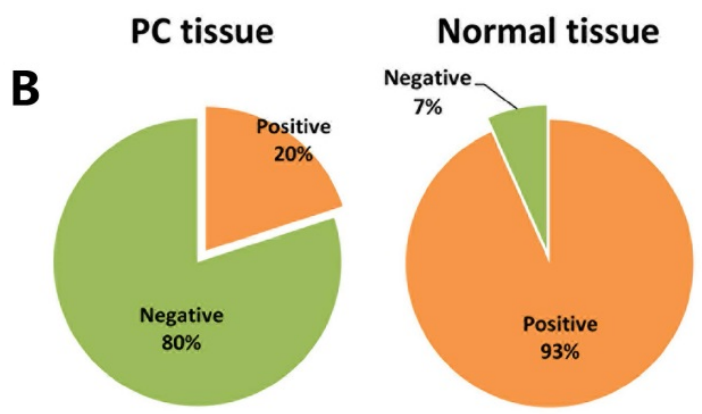

Staining instensity of RBM5
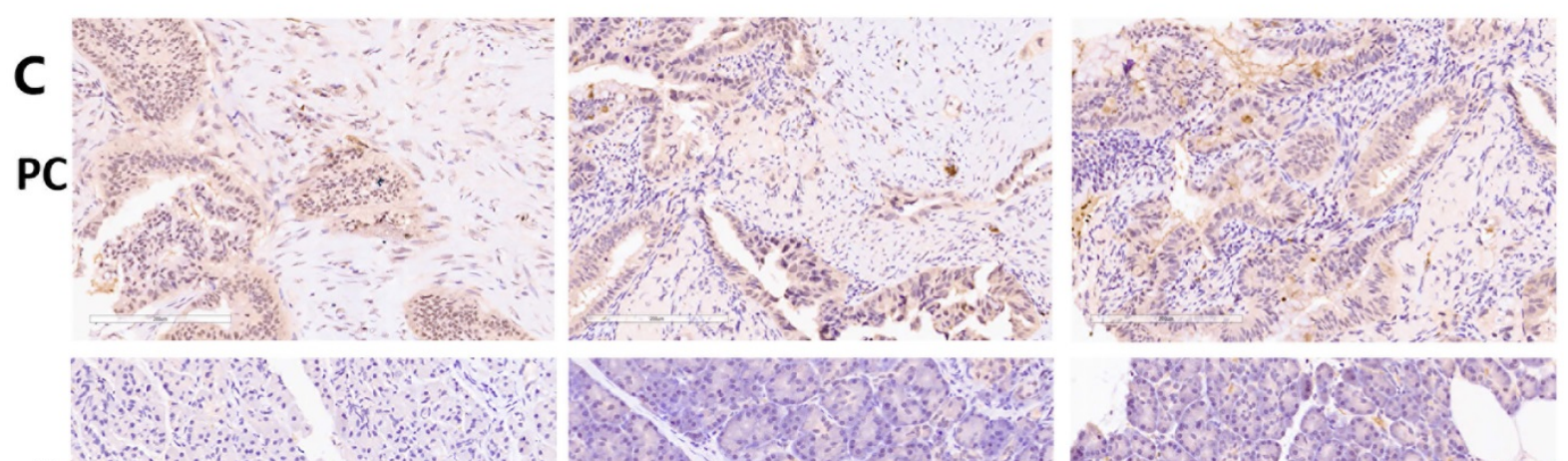

$\mathbf{N}$
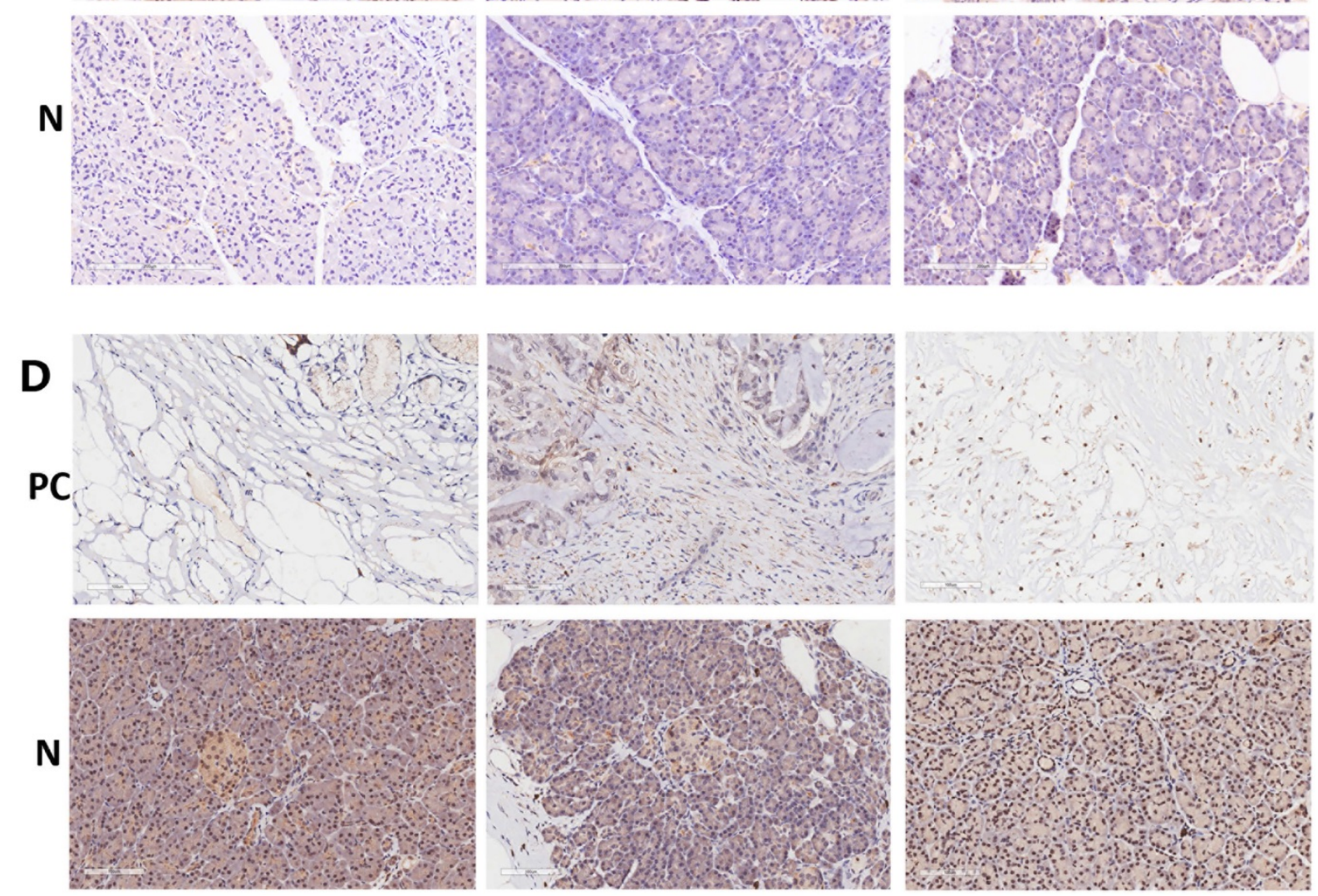

Figure 8. Representative images for immunostaining of splicing factors ESRP I and RBM5. (A) Pie chart reports the numbers of PC tissues versus normal tissues with 'positive' or 'negative' ESRPI staining. (B) Pie chart reports the numbers of PC tissues versus normal tissues with 'positive' or 'negative' RBM5 staining. (C) Immunostaining for ESRPI of PC tissues and normal tissues (x200). (D) Immunostaining for RBM5 of PC tissues and normal tissues (x200).

Alternative splicing events affecting pancreatic adenocarcinoma have also been found in many confirmatory studies. For example, two alternative splicing isoforms of RHAMM are RHAMMA and RHAMMB. RHAMMB is significantly upregulated in pancreatic adenocarcinoma liver metastasis, which promotes PNET metastasis via EGFR signalling, whereas RHAMMA does not [27]. Compared to WT-MUC4, MUC4/X enhances panc-1 cell proliferation, invasion and adhesion and promotes pancreatic tumourigenesis via the integrin- $\beta 1 / F A K /$ ERK signalling pathway [28]. In the study of pancreatic adenocarcinoma treatment strategies, it was found that PKM2 splicing and expression are 
functionally related to the resistance of gemcitabine and cisplatin. Switching splicing of PKM1 by ASO transfection can enhance cell drug sensitivity [29]. AS events of FGFR-2, SPAR, COL6A3 in pancreatic adenocarcinoma have also been previously reported [30-32], and an increase in AS of the KLF6 cancer suppressor gene is associated with pancreatic adenocarcinoma prognosis and tumour grade [33]. Therefore, a comprehensive understanding of the AS model is critical to the future treatment strategy for cancer. The emergence of RNA-Seq technology and the cancer genome database has become a new tool for studying AS events in pancreatic adenocarcinoma, helping to identify new therapeutic and molecular targets for pancreatic adenocarcinoma [34, 35].

In this study, we compared differentially spliced AS transcripts of pancreatic adenocarcinoma tissues and non-tumour tissues and genome-wide survival-related AS events in 171 patients with pancreatic adenocarcinoma. A total of 45,313 AS events were identified, including 1546 differentially alternative splicing events, suggesting that alternative splicing is a common process in pancreatic adenocarcinoma. Specifically, we analysed seven types of splicing patterns of AA, AD, AP, AT, ES, RI, and $\mathrm{ME}$ and found that ES is the most important splicing method, which may also be responsible for the biological activity and protein complication of pancreatic adenocarcinoma [36]. Furthermore, we found that genes for prognosis-related AS events are involved in multiple metabolic pathways involved in cancer cell biology and interact closely with each other. And by multivariate Cox regression analysis, 19 DEAS events were found to be associated with the prognosis of pancreatic adenocarcinoma. The prognostic predictors established in the study performed well in PAAD with a five-year overall survival rate of 0.946 , representing a potentially reliable predictor of AS events in PAAD patients. Prognostic genes include LAS1L, SUPT4H1, FAM72A, C11orf31 and MRPS22, which play key roles in the biological mechanisms of cancer. At the same time, these prognostic genes may also participate in the process of cancer biology through means other than alternative splicing. For example, LAS1L and SENP3, as components of the MLL1-WDR5 super complex, regulate pancreatic adenocarcinoma gene transcription by affecting chromatin remodelling [37]. MRPS22 is thought to be a potential driver involved in DNA replication, mismatch repair, p53 signalling pathway and cancer-associated signalling pathways [38]. C11orf31 is involved in the regulation of drug resistance and cellular reactivity of ascorbic acid in a variety of cancer cells [39].
At a deeper level, we identified 15 splicing factors associated with the prognosis of PAAD, including the universal splicing factor SFs and the hnRNP family [40, 41]. A combination of splicing factor expression and alternative splicing events and related networks are constructed to reveal the underlying mechanisms of the AS pathway. We found a close relationship between 15 splicing factors and 17 splicing genes, while the splicing factors ESRP1 and RBM5 performed well in the network. Studies have shown that ESPR1 and RBM5 play an important role in the event of alternative splicing in cancer [42, 43], and the results of GESA also show that ESRP1 and RBM5 are significantly enriched in the spliceosome pathway. In addition, evidence from experiments shows the interaction between ESRP1 and hnRNPM is related to EMT and breast cancer subtyping [44]. ESRP1 can regulate the expression of FGFR-2 isoform FGFR-2IIIb, attenuates cell growth, migration, invasion and metastasis, and is a prognostic factor for pancreatic adenocarcinoma [45]. Subsequent analysis of subgroups of patients with different clinical variables in the TCGA cohort showed that ESRP1 and RBM5 parts showed prognostic differences, but this does not mean that the results are biased, which may be due to data bias, population susceptibility and cancer heterogeneity. In short, we used databases such as TCGA, GEO, GTEx and immunohistochemical experiment to focus on analyzing the complications of alternative splicing on pancreatic cancer, and believe that we can have an in-depth understanding of the regulatory mechanisms of PAAD patients.

In summary, we found differential splicing of AS events between PAAD and normal tissues and established a model to demonstrate that survival-associated AS features can be used to predict prognosis in PAAD patients. The SF-AS network suggests a new potential mechanism in the carcinogenesis of PAAD, and the splicing factors ESRP1 and RBM5 played an important role in the PAAD-associated AS events. The systematic study of alternative splicing mechanisms provides a new direction for the treatment strategy of pancreatic adenocarcinoma.

\section{Abbreviations}

AS: Alternative splicing; PAAD: Pancreatic Adenocarcinoma; DEAS: Differentially Expressed Alternative Splicing Events; TCGA: The Cancer Genome Atlas; PSI: Splicing Percentage Index; ES: Exon Skip; ME: Mutually Exclusive Exons; RI: Retained Intron; AP: Alternate Promoter; AT: Alternate Terminator; AD: Alternate Donor; AA: Alternate Acceptor; OS: Overall Survival; ROC: 
Receiver Operating Characteristic; KEGG: Kyoto Encyclopedia of Genes and Genomes Pathway; GEO: Gene Expression Omnibus database; GTEx: Genotype-Tissue Expression.

\section{Supplementary Material}

Supplementary figures and tables. http://www.jcancer.org/v12p3164s1.pdf

\section{Acknowledgements}

This study was supported by the Project of National Natural Science Foundation of China (81902385), the Project of Suzhou People's Livelihood Science and Technology (sys2018037), the Jiangsu Provincial Medical Youth Talent (QNRC2016734), the Six Talent Peaks Project in Jiangsu Province (WSW-059), and the Project of Medical Research of Jiangsu Province (Y2018094, H2018056).

\section{Ethics Committee Approval and Patient Consent}

The study was in accordance with the ethical standards formulated in the Helsinki Declaration and was approved by the Science and Technology Ethics Committee of the First Affiliated Hospital of Soochow University. All patients enrolled completed the informed consent form.

\section{Competing Interests}

The authors have declared that no competing interest exists.

\section{References}

1. Siegel RL, Miller KD, Jemal A. Cancer statistics, 2018. CA: a cancer journal for clinicians. 2018; 68: 7-30.

2. Ngamruengphong $S$, Canto MI. Screening for pancreatic cancer. The Surgical clinics of North America. 2016; 96: 1223-33.

3. Rahib L, Smith BD, Aizenberg R, Rosenzweig AB, Fleshman JM, Matrisian LM. Projecting cancer incidence and deaths to 2030: The unexpected burden of thyroid, liver, and pancreas cancers in the United States. Cancer research. 2014; 74: 2913-21.

4. Sultana A, Smith CT, Cunningham D, Starling N, Tait D, Neoptolemos JP, et al. Systematic review, including meta-analyses, on the management of locally advanced pancreatic cancer using radiation/combined modality therapy. British journal of cancer. 2007; 96: 1183-90.

5. Ge Y, Porse BT. The functional consequences of intron retention: Alternative splicing coupled to NMD as a regulator of gene expression. BioEssays : news and reviews in molecular, cellular and developmental biology. 2014; 36: 23643.

6. Mao S, Li Y, Lu Z, Che Y, Huang J, Lei $Y$, et al. PHD finger protein 5A promoted lung adenocarcinoma progression via alternative splicing. Cancer medicine. 2019; 8: 2429-41.

7. Munkley J, Livermore K, Rajan P, Elliott DJ. RNA splicing and splicing regulator changes in prostate cancer pathology. Human genetics. 2017; 136: 1143-54.

8. Yao RW, Wang Y, Chen LL. Cellular functions of long noncoding RNAs. 2019; 21: 542-51.

9. Zhou X, Wang R, Li X, Yu L, Hua D, Sun C, et al. Splicing factor SRSF1 promotes gliomagenesis via oncogenic splice-switching of MYO1B. The Journal of clinical investigation. 2019; 129: 676-93.

10. Wang Q, Wang Y, Liu Y, Zhang C, Luo Y, Guo R, et al. U2-related proteins CHERP and SR140 contribute to colorectal tumorigenesis via alternative splicing regulation. 2019; $145:$ 2728-39.

11. Yuan JH, Liu XN, Wang TT, Pan W, Tao OF, Zhou WP, et al. The MBNL3 splicing factor promotes hepatocellular carcinoma by increasing PXN expression through the alternative splicing of lncRNA-PXN-AS1. Nature cell biology. 2017; 19: 820-32
12. de Santiago I, Yau C, Heij L, Middleton MR, Markowetz F, Grabsch HI, et al. Immunophenotypes of pancreatic ductal adenocarcinoma: Meta-analysis of transcriptional subtypes. International journal of cancer. 2019; 145: 1125-37.

13. Seth S, Li CY, Ho IL, Corti D, Loponte S, Sapio L, et al. Pre-existing functional heterogeneity of tumorigenic compartment as the origin of chemoresistance in pancreatic tumors. Cell reports. 2019; 26: 1518-32.e9.

14. Li Y, Sun N, Lu Z, Sun S, Huang J, Chen Z, et al. Prognostic alternative mRNA splicing signature in non-small cell lung cancer. Cancer letters. 2017; 393: 4051.

15. Yang $\mathrm{Q}$, Zhao J, Zhang W, Chen D, Wang Y. Aberrant alternative splicing in breast cancer. Journal of molecular cell biology. 2019: mjz033.

16. Zong Z, Li H, Yi C, Ying H, Zhu Z, Wang H. Genome-wide profiling of prognostic alternative splicing signature in colorectal cancer. Frontiers in oncology. 2018; 8: 537.

17. Chen F, Chen J, Yang L, Liu J, Zhang X, Zhang Y, et al. Extracellular vesicle-packaged HIF-1alpha-stabilizing lncRNA from tumour-associated macrophages regulates aerobic glycolysis of breast cancer cells. 2019; 21: 498-510.

18. Giulietti M, Piva F, D'Antonio M, D'Onorio De Meo P, Paoletti D, Castrignano T, et al. SpliceAid-F: A database of human splicing factors and their RNA-binding sites. Nucleic acids research. 2013; 41: D125-D31.

19. Liu B, Jia Y, Cao Y, Wu S, Jiang H, Sun $X$, et al. Overexpression of Phosphoserine Aminotransferase 1 (PSAT1) Predicts Poor Prognosis and Associates with Tumor Progression in Human Esophageal Squamous Cell Carcinoma. Cellular physiology and biochemistry : international journal of experimental cellular physiology, biochemistry, and pharmacology. 2016; 39: 395-406.

20. Oltean S, Bates DO. Hallmarks of alternative splicing in cancer. Oncogene. 2014; 33: 5311-8

21. Song Q, Yi F, Zhang Y, Jun Li DK, Wei Y, Yu H, et al. CRKL regulates alternative splicing of cancer-related genes in cervical cancer samples and HeLa cell. BMC cancer. 2019; 19: 499.

22. Buoso E, Ronfani M, Galasso M, Ventura D, Corsini E, Racchi M. Cortisol-induced SRSF3 expression promotes GR splicing, RACK1 expression and breast cancer cells migration. Pharmacological research. 2019; 143:17-26.

23. Sakuma K, Sasaki E, Kimura K, Komori K, Shimizu Y, Yatabe Y, et al. HNRNPLL, a newly identified colorectal cancer metastasis suppressor, modulates alternative splicing of CD44 during epithelial-mesenchymal transition. Gut. 2018; 67: 1103-11.

24. Coomer AO, Black F, Greystoke A, Munkley J, Elliott DJ. Alternative splicing in lung cancer. Biochimica et Biophysica Acta (BBA) - Gene Regulatory Mechanisms. 2019: S1874-9399(18)30528-5.

25. Wang X, Guo J, Che X, Jia R. PCBP1 inhibits the expression of oncogenic STAT3 isoform by targeting alternative splicing of STAT3 exon 23. International journal of biological sciences. 2019; 15: 1177-86.

26. Shao Y, Chong W, Liu X, Xu Y, Zhang H, Xu Q, et al. Alternative splicing-derived intersectin1-L and intersectin1-S exert opposite function in glioma progression. Cell Death and Disease. 2019; 10: 431.

27. Choi S, Wang D, Chen X, Tang LH, Verma A, Chen Z, et al. Function and clinical relevance of RHAMM isoforms in pancreatic tumor progression. Molecular cancer. 2019; 18: 92.

28. Jahan R, Macha MA, Rachagani S, Das S, Smith LM, Kaur S, et al. Axed MUC4 (MUC4/X) aggravates pancreatic malignant phenotype by activating integrin-beta1/FAK/ERK pathway. Biochimica et biophysica acta Molecular basis of disease. 2018; 1864: 2538-49.

29. Calabretta S, Bielli P, Passacantilli I, Pilozzi E, Fendrich V, Capurso G, et al. Modulation of PKM alternative splicing by PTBP1 promotes gemcitabine resistance in pancreatic cancer cells. Oncogene. 2016; 35: 2031-9.

30. Arafat H, Lazar M, Salem K, Chipitsyna G, Gong Q, Pan TC, et al. Tumor-specific expression and alternative splicing of the COL6A3 gene in pancreatic cancer. Surgery. 2011; 150: 306-15.

31. Matsuda $Y$, Yoshimura $H$, Suzuki $T$, Uchida E, Naito Z, Ishiwata T. Inhibition of fibroblast growth factor receptor 2 attenuates proliferation and invasion of pancreatic cancer. Cancer science. 2014; 105: 1212-9.

32. Viloria K, Munasinghe A, Asher S, Bogyere R, Jones L, Hill NJ. A holistic approach to dissecting SPARC family protein complexity reveals FSTL-1 as an inhibitor of pancreatic cancer cell growth. Scientific reports. 2016; 6: 37839.

33. Hartel M, Narla G, Wente MN, Giese NA, Martignoni ME, Martignetti JA, et al. Increased alternative splicing of the KLF6 tumour suppressor gene correlates with prognosis and tumour grade in patients with pancreatic cancer. European Journal of Cancer. 2008; 44: 1895-903.

34. Park E, Pan Z, Zhang Z, Lin L, Xing Y. The expanding landscape of alternative splicing variation in human populations. American journal of human genetics. 2018; 102: 11-26.

35. Sharma V, Nandan A, Singh H, Agarwal S, Tripathi R, Sinha DN, et al. Events of alternative splicing in head and neck cancer via RNA sequencing - an update. BMC genomics. 2019; 20: 442 .

36. Kim HK, Pham MHC, Ko KS, Rhee BD, Han J. Alternative splicing isoforms in health and disease. Pflügers Archiv. 2018; 470: 995-1016.

37. Kashiwaya K, Nakagawa H, Hosokawa M, Mochizuki Y, Ueda K, Piao L, et al. Involvement of the tubulin tyrosine ligase-like family member 4 polyglutamylase in PELP1 polyglutamylation and chromatin remodeling in pancreatic cancer cells. Cancer research. 2010; 70: 4024-33. 
38. Lazar V, Suo C, Orear C, van den Oord J, Balogh Z, Guegan J, et al. Integrated molecular portrait of non-small cell lung cancers. BMC medical genomics. 2013; 6: 53.

39. Ooko E, Kadioglu O, Greten HJ, Efferth T. Pharmacogenomic characterization and isobologram analysis of the combination of ascorbic acid and curcumin-two main metabolites of curcuma longa-in cancer cells. Frontiers in pharmacology. 2017; 8: 38 .

40. Kedzierska H, Piekielko-Witkowska A. Splicing factors of SR and hnRNP families as regulators of apoptosis in cancer. Cancer letters. 2017; 396: 53-65.

41. Xu X, Yang J, Zhou W, Wang L, Lu Q, Wang X, et al. Genetic variations within alternative splicing associated genes are associated with breast cancer susceptibility in Chinese women. Gene. 2019; 706: 140-5.

42. Lee S, Cieply B, Yang Y, Peart N, Glaser C, Chan P, et al. Esrp1-Regulated Splicing of Arhgef11 Isoforms Is Required for Epithelial Tight Junction Integrity. Cell reports. 2018; 25: 2417-30.e5.

43. Sun Y, Bao Y, Han W, Song F, Shen X, Zhao J, et al. Autoregulation of RBM10 and cross-regulation of RBM10/RBM5 via alternative splicing-coupled nonsense-mediated decay. Nucleic acids research. 2017; 45: 8524-40.

44. Harvey SE, Xu Y, Lin X, Gao XD, Qiu Y, Ahn J, et al. Coregulation of alternative splicing by hnRNPM and ESRP1 during EMT. RNA (New York, NY). 2018; 24: 1326-38

45. Ueda J, Matsuda Y, Yamahatsu K, Uchida E, Naito Z, Korc M, et al. Epithelial splicing regulatory protein 1 is a favorable prognostic factor in pancreatic cancer that attenuates pancreatic metastases. Oncogene. 2014; 33: 4485-95. 